\title{
E-learning and intelligent content adaptation: an integrated approach
}

\section{Phivos Mylonas*, Paraskevi Tzouveli and Stefanos Kollias}

Department of Computer Science,

Image, Video and Multimedia Laboratory,

School of Electrical and Computer Engineering,

National Technical University of Athens,

PC GR 157 73, Zographou Campus, Athens, Greece

E-mail: fmylonas@image.ntua.gr_E-mail: tpar@image.ntua.gr

E-mail: stefanos@cs.ntua.gr

${ }^{*}$ Corresponding author

\begin{abstract}
It is a common fact that modern e-learning schemes lack educational content representation and user personalisation. In this framework, automated extraction of user profiles, to be used in an e-learning content offering system, forms an interesting and important problem. In this approach we present the design and implementation of such a profile-based system, by which content is matched to its environmental context, so that it can be adapted to its user's needs and capabilities. Current effort extends previous work on profile extraction via clustering techniques and on integrated e-learning systems. It relies on the fundamental IEEE e-learning model, suitably adapted to reflect and focus on profiling aspects of the system.
\end{abstract}

Keywords: web-based education; e-learning; personalised education systems; clustering-based profiling methods.

Reference to this paper should be made as follows: Mylonas, Ph., Tzouveli, P. and Kollias, S. (2007) 'E-learning and intelligent content adaptation: an integrated approach', Int. J. Continuing Engineering Education and Life-Long Learning, Vol. x, No. x, pp.xxx-xxx.

Biographical notes: Phivos Mylonas, MSc (Computer Science), is currently a researcher by the Image, Video and Multimedia Laboratory, School of Electrical and Computer Engineering, Department of Computer Science of the National Technical University of Athens, Greece. He obtained his Diploma in Electrical and Computer Engineering from the National Technical University of Athens in 2001, his Master of Science in Advanced Information Systems from the National and Kapodestrian University of Athens in 2003 and is currently pursuing his $\mathrm{PhD}$ Degree at the former University. His research interests lie in the areas of information retrieval, knowledge management, personalisation, e-learning, context, ontologies and software development.

Paraskevi Tzouveli is currently a researcher by the Image, Video and Multimedia Laboratory, School of Electrical and Computer Engineering, Department of Computer Science of the National Technical University of Athens, Greece. She obtained the Diploma in Electrical and Computer Engineering from the National Technical University of Athens in 2001 and is currently pursuing the $\mathrm{PhD}$ Degree at the same University. Her research interests lie in the areas of e-learning systems, ontologies, image and video analysis and software development. 
Stefanos Kollias is full Professor of National Technical University of Athens, Greece and Director of the Image, Video and Multimedia Systems Lab. $\mathrm{He}$ received his $\mathrm{PhD}$ in Signal Processing from the Computer Science Division, National Technical University of Athens. He has published more than 200 papers in Internationals Journals and Conferences. He is reviewer of more than 25 International Journals, reviewer of the Greek Secretariat of Research and Technology since 1989 and reviewer of the European Commission since 1996, in the Telematics, Esprit and IST Programme.

\section{Introduction}

The nature and structure of our era, dominated by rapid information exchange and instant worldwide communication capabilities, has significant impact on education and the way it evolves. In an epoch where everyone and everything continuously changes, education itself could not stay passive and unconcerned; all traditional teaching techniques are revisited and re-evaluated and new or sometimes radical ones are introduced (Commission of European Communities, 2000). Above all, the Internet plays a significant role in all fields of education, contributing the most to the educational procedures (Rosenberg, 2002). As a result, Internet-oriented applications arise in the aid of educational needs, trying to close the gap between traditional educational techniques and the new trends of future, technology-blended education.

The impact of Information and Communications Technology (ICT) in such a task has become more and more evident in learning and teaching at all levels of education (Karagiannidis et al., 2002). E-learning is unquestionably the revolutionary new way to empower a workforce with the skills and knowledge it needs. Towards that goal, during the last years, e-learning systems were developed in the means of static software applications, lacking in educational multimedia environments and personalised capabilities and without any interest given to the real users input and feedback (Sullivan, 1998; Varenne, 2001).

In this work, which was conducted in the framework of the Leonardo SPERO project (2001-2004), we present a novel method for gathering information and estimating the ICT level of learners in all fields of education. This is achieved through a web-based user-friendly interface that takes into consideration personalised, profile-based schemes. The latter has been designed to enable learners to gracefully increase their ICT knowledge and provide them with credible information and feedback, such as suitably selected e-courses and multimedia educational content.

The structure of this work is as follows: In Section 2, the overall architecture design of the SPERO system is introduced; its basic corresponding groups and components and its notion of e-questionnaires. Afterwards, a short reference to the IEEE e-learning model is presented, current approach's adaptation is analysed and the additional features provided by it are explained. In Section 3, we begin by tackling the problem of the learner profile creation, followed by issues concerning the initial static profile extraction procedures. All of the above are used as the main feedback source for the forthcoming intelligent clustering profiling procedure, which is presented in Section 4. In the same section a description of the utilised clustering algorithm is provided, together with experimental results on the clustering-based profiling scheme. Next, Section 5 describes 
the general context for this work, dealing with the educational content offering of the system and briefly presenting its categorised e-courses and operational examples of use. Finally, in Section 6, we present our concluding remarks and future work.

\section{System's architecture}

In the effort of designing, implementing and evaluating a novel, integrated e-learning system, the first step to consider is the definition of its basic architecture. The general architecture design of SPERO is shown in Figure 1.

Figure 1 SPERO system architecture

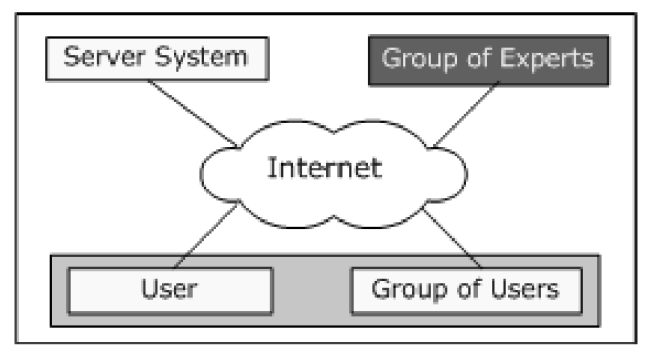

Three main networked components can be identified and they are:

- the group of the system's users

- the group of system's experts, who play a key role in the initialisation of the personalisation process; this group includes teachers, experts in e-learning, data analysts, psychologists and software engineers

- the actual server system, which includes all hardware and software needed to establish a 2-tier system core (Skordalakis, 1991).

The first group includes every kind of teacher working either for general education or for the Special Education sector. Eventually, expanding the system's architecture, a user could be identified as any learner, whether a student, teacher or employee. The second group's role is crucial in the personalisation process of the SPERO system, because it defines the initial set of specifications and limitations of the end-users' profiles, which justifies the variety of people comprising it. The third group includes all hardware and software that enables a web-server to be active, as well as efficient and robust. All distinct SPERO web applications (SPERO e-questionnaires; SPERO statistics; SPERO profiles) together with an underlying Relational Database Management System to support profiling and user information are included in this configuration.

Moreover, one of the basic interaction components of SPERO and the mean of communication with its end-users is formed by the so-called e-questionnaire (SPERO e-questionnaires). SPERO experts have designed and illustrated two groups of e-questionnaires: The first group contains questions about school units in order to collect general details about them. The second group contains questions about teachers' ICT background. The questions, which are addressed to the teachers, are intended to collect information about teachers' educational background, as well as their background in ICT. 
In addition, information concerning teachers' opinions about pedagogical utilisation of ICT and the amount of using ICT in teaching procedure is also extracted. As already mentioned, learners could either be teachers or students, however both of them are in great need of ICT: on the one hand, teachers mainly because their role is continuously evolving and demanding new formation and on the other hand students, because of their need to have distance e-courses in the field of ICT. Each questionnaire is divided into several subsections, a portion of which is depicted in Figure 2. The presented sub-questionnaire collects information about general teachers' educational background, as well as their background in ICT.

Figure 2 Part of teachers' questionnaire

\begin{tabular}{|c|c|c|}
\hline \multicolumn{3}{|c|}{ Teacher Questionnaire } \\
\hline \multicolumn{3}{|c|}{ A. Pedagogical Utilisation of Information Technologies } \\
\hline \multirow{2}{*}{\multicolumn{3}{|c|}{ 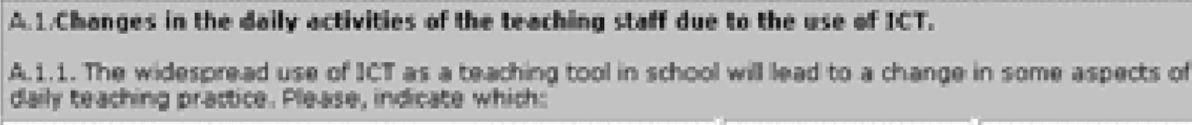 }} \\
\hline & & \\
\hline & Mostly & Farely \\
\hline $\begin{array}{l}\text { A change of attitude of the thaching staff will be } \\
\text { necessary }\end{array}$ & 0 & 0 \\
\hline $\begin{array}{l}\text { New leaming actruitien with the studentif will have be } \\
\text { designed and implemented }\end{array}$ & 0 & 0 \\
\hline It will not affect other leaming activities & 0 & 0 \\
\hline
\end{tabular}

SPERO e-questionnaires are developed in the framework of conducting a European survey. Consequently, they are translated in eight European languages and these translations are stored within SPERO's Relational Database Management System. Software has been developed for automatic presentation of e-questionnaires in every one of these eight languages. Moreover, the e-questionnaire is used to estimate the ICT level of individual users, by using the calculated user's profile categorisations, which are automatically extracted in the following by the SPERO software. More than one learning resources (e-courses and educational material) are selected by experts, up to one for each of the distinct collaborative user profiles categories. The set of e-questionnaires is used for ICT level estimation in the framework of the distance-learning architecture that is presented in Figure 3. 
Figure 3 IEEE learning system entities

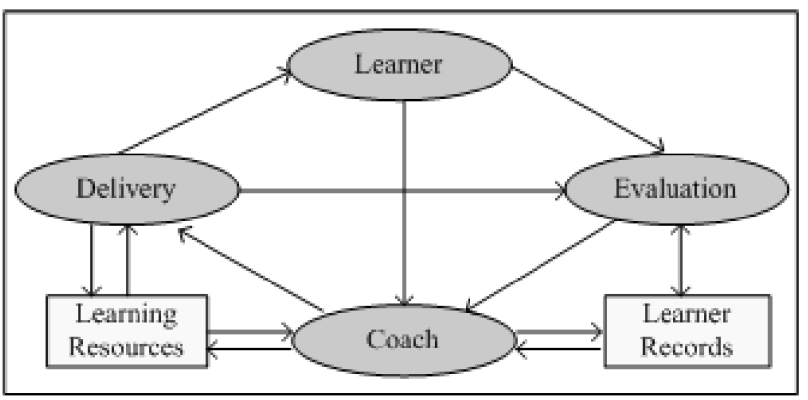

In this work we attempt to extract learner profiles through the evaluation entity of the above architecture, proposed by the IEEE Reference Model (WG1 LTSA) of the Learning Technology Standards Committee (IEEE LTSC, 2002). This standard covers a wide range of systems, such as learning technology, education and training technology, computer-based training, computer assisted instruction, intelligent tutoring, and is pedagogically neutral, content-neutral, culturally neutral, and platform-neutral. However, in this generic approach to e-learning systems, a system's ability to adapt its operation to the user is not defined, although an evaluation process exists. Aiming at extracting learner profiles through this entity, we are proposing the replacement of the IEEE standard Evaluation entity, with the novel Re-evaluation entity, which, additionally, is strongly related to two new entities: the E-survey entity for gathering statistical information and the Profile Database entity dealing with all learners' profiles. A schematic diagram of the proposed replacement is presented in Figure 4, the replaced learning components are shown in Figure 5, whereas a detailed description of the above concepts can be found in Mylonas et al. (2004a).

Figure 4 Proposed replacement of Evaluation entity

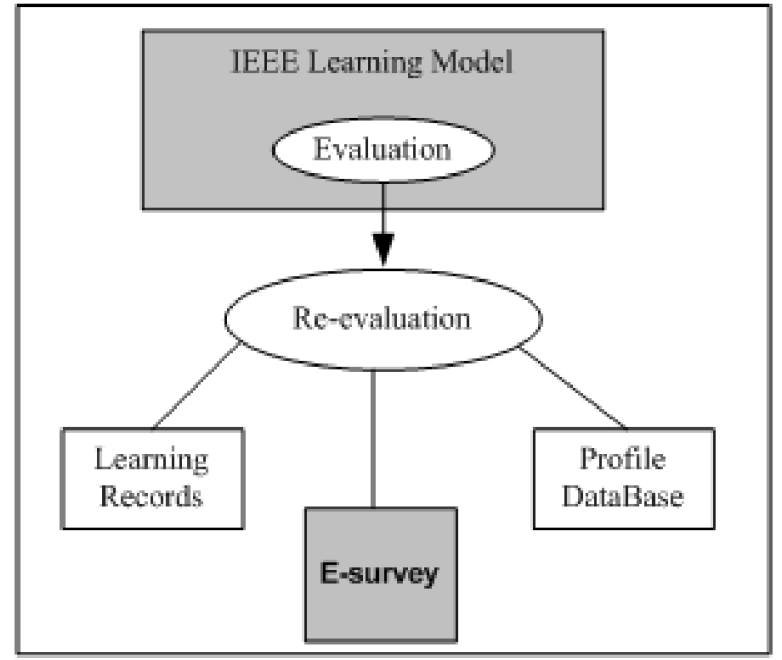


Figure 5 Proposed e-learning system after replacement of Evaluation entity

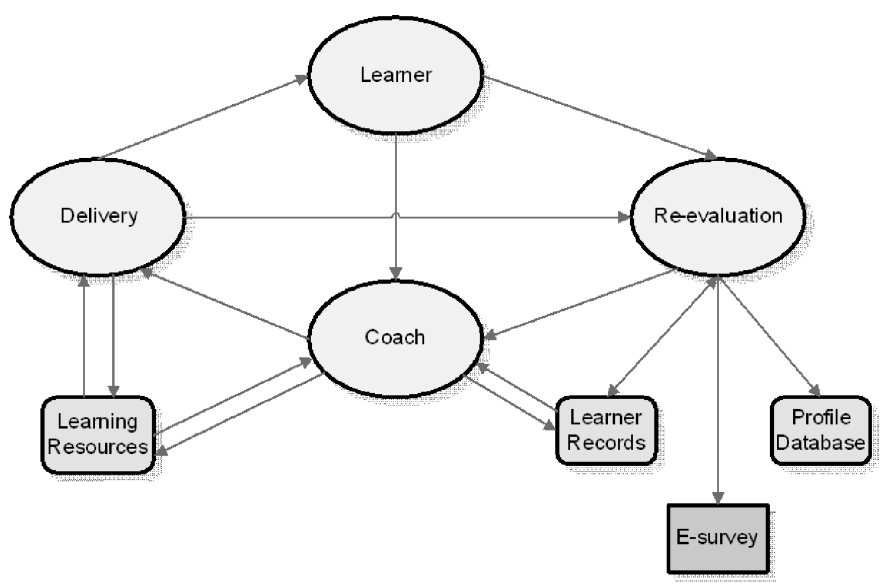

In order to assist the profiling process, the need for this re-evaluation step is essential; the usage of an appropriate e-questionnaire is considered necessary in order to collect user input data and build a large, reliable ground truth, basing profiling information on top of them. For this purpose, experts designed and illustrated an e-questionnaire (SPERO E-questionnaires) which collects information about learners' ICT background, learners' opinions about pedagogical utilisation of ICT and the amount of using ICT in teaching procedure. Additionally, software has been developed to allow e-surveys to be conducted based on users' answers.

As a result, the core of the system relies on this replaced Evaluation entity. The latter forms an independent personalisation subsystem, where user- profiling information is extracted, according to statistics gathered from the e-questionnaire database and the e-survey. Delivery of educational content is then possible, based on the results of the profiling procedure, providing personalised views to the system's end-users and taking into consideration their particular ICT levels of education and needs.

\section{Profiling initialisation}

At this point, a brief presentation of the system's personalisation subsystem is essential. It is the intended nature of SPERO that dictates utilisation of two profiling approaches; both of them look very different in the beginning, but they are combined at a later phase. One approach is followed at the initial stage of constructing the profiling ground truth and is characterised by a static profiling mechanism, whereas the second approach exploits results provided by the previous one towards dynamic extraction of current and future SPERO users. First step is materialised through a fixed mapping of obtained user input to specific types of user profiles explained in the following within this section. Second step is acting on top of the first one and is founded on application of a clustering technique (Mitchell, 1997). The latter is presented in the next section. Accordingly, the corresponding dataset was divided into two parts, one primary utilised by experts during the first step and another utilised by the automated clustering methodology. 
Let us illustrate the functionality of the initial profile representation, which is based on the static profiling mechanism. Experts, based on experience and intuition, define a set of three user characterisations, forming a static profiling representation. This step forms a pre-processing task performed by hand, combined with statistical analysis and later cross-correlation selection of features, which indicates the most important ones that were considered in the following. It is considered to be extremely reliable and accurate, as it was the result of three years of intensive collaboration among experts from eight European countries that examined this through questionnaires in national and cross-national analysis. These initial characterisations are also utilised at a later stage, during the intelligent profiling process, providing a rock-solid point of reference and although they are thought to be static, they are actually generated automatically from the system. Their basis is information provided by the users' input data, obtained from the e-questionnaires (SPERO E-questionnaires). In this case, personalisation was needed in order to aid with the ultimate educational content offered by the system; this was successful, based on the electronic mining of knowledge gathered from the system's questionnaires subsystem.

The profiling mechanism creates updates and uses system's user profiles, matching specific e-questionnaires question triggers, to particular identified patterns. The profile model's design facilitates both the process of using user preferences in profile creation, as well as the process of preference tracking throughout the whole profiling procedure. Furthermore, it is designed in a way that allows for the automated extraction of user profiles, based on these preferences and the users' input history. This model forms an initial static version of the user profile denoted by UserProfile and presented in Figure 6.

Figure 6 Structure of the UserProfile

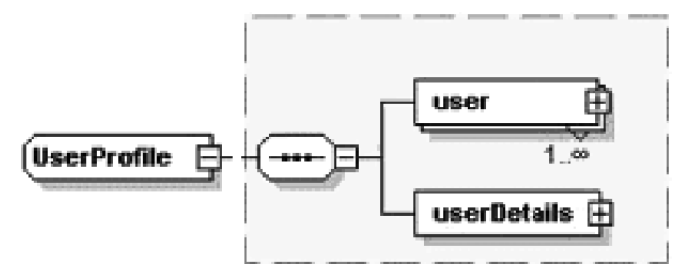

As seen in the figure, the main abstract structure of UserProfile compound type contains two elements. The first user, stores information about the user's history, while the second, userDetails, stores the user preferences. As the initial profiling process instantiates, all user profiles are stored within a single, central mapping structure, whose abstract model is presented in Figure 7. The UserProfile is mapped against information retrieved either from the e-questionnaire itself, or directly from the input of the users. The first element, QuestionId holds all the information required for identifying the underlying e-questionnaire question, as well as its type, aiming at better understanding and fitting of the currently generated profile. The second element, UserInput contains user data related information, such as the user's answers. Both, the sequences of QuestionId and UserInput elements denote the existence of large amount of different system's e-questionnaires, questions and users' input data. 
The core of this methodology is summarised in the following step of weights association, performed according to the following guidelines: Once a user answers a question of the input e-questionnaire, a relevance degree is associated with it and adjusted to her or his specific QuestionId element, and thus also propagated to the UserProfile element. As more and more answers from the end user enter the UserProfile structure, additional relevance degrees are registered to the corresponding QuestionID elements. Depending on the particular question, as well as the part of the e-questionnaire that this question belongs, different degrees are propagated. The latter is based on comparison of the provided numerical values with the range of values a-priori associated with the profiles. In order to better understand the underlying mapping structures, examples are presented in Figures 8-10, derived from the group of experts directly from the e-questionnaires:

Figure 7 Mapping structure of UserProfiles

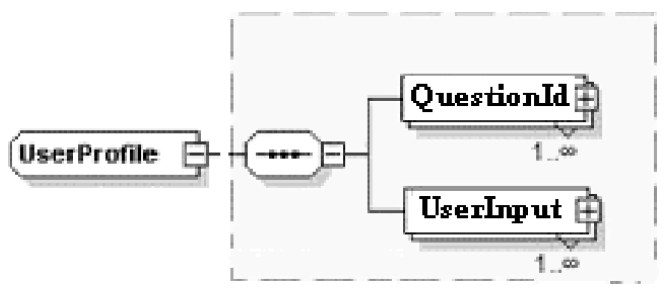

Figure 8 Static profile mapping example (1st part of e-questionnaire)

\begin{tabular}{|l|c|}
\hline B.1.4.Teaching Experience & \\
\hline Under 5 years & Beginner \\
\hline 5 to 10 years & Advanced \\
\hline 11 to 20 years & Advanced / Expert \\
\hline Over 20 years & Expert \\
\hline
\end{tabular}

Figure 9 Static profile mapping example (2nd part of e-questionnaire)

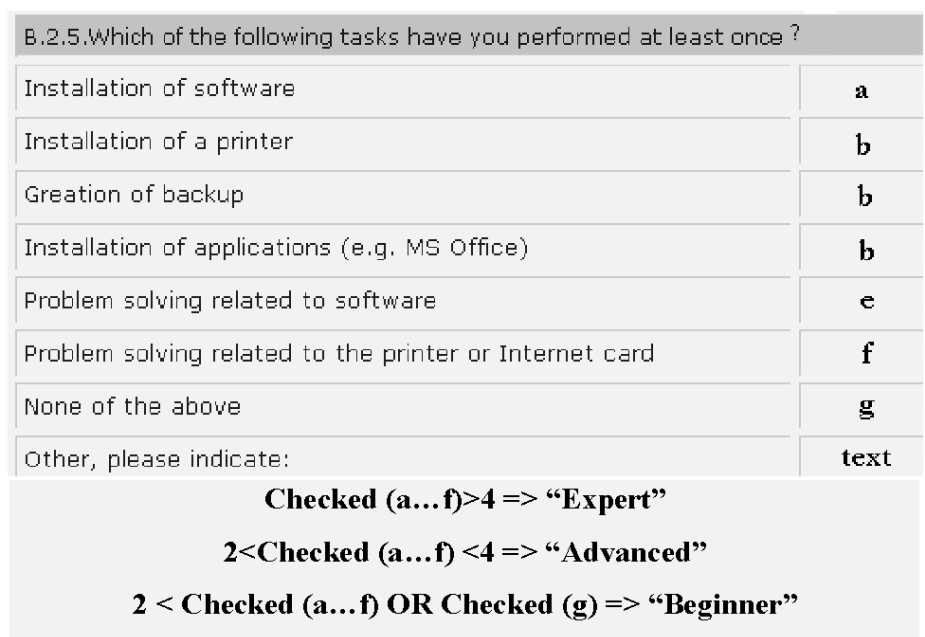


Figure 10 Static profile mapping example (3rd part of e-questionnaire)

B.3.4. How many hours a week, on awerage, do you use the Internet or educational software with your
students?
$\begin{aligned} & \text { General } \\ & \text { SEN } \\ & \text { if (General or/and SEN teaching hours) }>5 \text { " }=>\text { Expert" }\end{aligned}$

The e-questionnaire acts as an intermediate towards the information gathering process, and as the amount of the answered questions increases, the more entries are summed up in the 'UserProfile' structure. Thus, the overall process results in an aggregated weighted mapping of the end user to the specified profile, which is different for each user's answers and depends on their particular nature. This mapping is temporarily preserved and as the completion of the e-questionnaire is carried out, the above-mentioned weighted mappings are aggregated. In that manner, they continuously and dynamically change every user's profiling, until a final equilibrium profile state is achieved. Test bed experimental results within the SPERO project indicate that after an approximately $50 \%$ sample of questions has been answered, it is possible for the system to balance to a solid, static, initial user profile with great confidence. As a result, the entire pre-processing task is governed by great accuracy and reliability, although in general it is difficult to handle in such cases, where data are characterised by numerous measurable features like answers to e-questionnaires. This is the case when multiple independent features characterise data, and thus more than one meaningful similarity or dissimilarity measures can be defined. A common approach to the problem is the lowering of input dimensions (Kohavi and Sommerfield, 1995), which may be accomplished by ignoring some of the available features/answers, and is the one followed herein. Additionally, a statistical cross-correlation analysis of the importance of features was also performed. For the sake of space we omit detailed presentation of the feature selection process, however the basic principle to be followed throughout this paper is that while we expect elements of a given meaningful set of e-questionnaires to have random distances from one another according to most features, we expect them to have small distances according to the features that relate them. We rely on this difference in distribution of distance values in order to identify the context of a set of elements, i.e., the subspace in which the set is best defined and provides the most meaningful results in terms of semantic clarity. The result of this process is a set of 44 meaningful e-questionnaire questions.

The final output of this process, following the application of the weights, is the extraction of a ' $1-1$ ' profile end -user relation. In that manner, each end user is classified to an initial, static profile that characterises his behaviour, his interests and his further treatment from the system. This particular profile characterisation forms the basis of the following intelligent clustering procedure, which includes the notion of profile extraction and integration within this system. In Figure 11 we present an indicative sample of the end-users' static profiling, extracted by previously analysed procedure within the SPERO system, according to the users' answers collected by the e-questionnaires. 
Figure 11 Initial static profiling mapping

\begin{tabular}{|c|c|c|c|c|}
\hline & User ID & Professional Development & Personal ICT Background & Teaching use of ICT \\
\hline 1. & 509 & begines & Unscacified & baginner \\
\hline 2 & 659 & bogirner & ungreacified & beginnar \\
\hline 3. & EOPT & ㅁapport & mant & beginner \\
\hline 4. & BOE: & unzpecified & beginner & baginnet \\
\hline 5. & 009 & expert & enoet & advanced \\
\hline 6. & B11 & expert & evoert & advanced \\
\hline 7 & Bi13 & exanart & enoest & advanced \\
\hline 8. & $\underline{\mathrm{B} 14}$ & eapart & expert & idvanced \\
\hline
\end{tabular}

\section{Clustering-based profiling}

At this point, experts are considered to have successfully labelled the first part of the dataset, provided initially as the ground truth of the profiling mechanism, by assigning profiles to each user according to the responses given to e-questionnaires. The next step to follow consists of applying intelligent clustering techniques to group similar profiles in the current as well as second part of the dataset. Traditional classification schemes are not applicable in this case and an intelligent clustering methodology is favoured. This step is necessary for the unsupervised operation of SPERO, where no manual labelling is available. Of course, at this phase various predefined profile schemes may arise and results are not bound to the so far manual characterisation of system's experts. In this manner, more than three profiles may be obtained and categorisation to the predominant ones need to be applied. The latter is necessary for us, in order to be able to compare results between the two phases and derive meaningful conclusions. After a small theoretical analysis of the proposed clustering technique, we present in detail the steps of the clustering algorithm for the problem at hand.

The core of the clustering data concept is to identify homogeneous groups of objects based on the values of their attributes. It is in general a difficult problem to tackle and is undoubtfully related to various scientific and applied fields (Commission of European Communities, 2000), especially when clustering is applied to user modelling (Paliouras et al., 2002; Smith, 2001). The problem gets more and more challenging, as input space dimensions become larger and feature scales are different from each other, as is the case in our system. In particular, a consideration of the original set of questions of the e-questionnaires as input space, results into a large number of 176 unique features to be taken into consideration when performing clustering on the user answers. The best way to go in this direction is to use a hierarchical clustering algorithm, which is able to tackle such a large scale of features (Duda et al., 2001; Mylonas et al., 2004b; Theodoridis and Koutroumbas, 1999). Although such a method does not demand the number of clusters as input, still it does not provide a satisfactory framework for extracting meaningful results. This is mainly owing to the 'curse of dimensionality' that dominates such an approach, as well as the inevitable initial error propagation and complexity along with data set size issues.

In order to increase the robustness and reliability of the whole clustering step of our system, the use of an unsupervised extension to hierarchical clustering in the means of feature selection was evident (Mylonas et al., 2004b). Using the results of the application 
of this clustering to a portion of the system's dataset in question is then refined and extended to the whole dataset. The performance of the proposed methodology is finally compared to the previous step of fixed clustering, using the predefined profile characterisations as a priori label information.

The general structure of such hierarchical clustering algorithms, which forms the structure of SPERO's clustering approach as well, is summarised in the following steps and presented analytically in Mylonas et al. (2004b):

1 Turn each input element into a singleton, i.e., into a cluster of a single element.

2 For each pair of clusters $c_{1}, c_{2}$ calculate their distance $d\left(c_{1}, c_{2}\right)$.

3 Merge the pair of clusters that have the smallest distance.

4 Continue at step 2, until the termination criterion is satisfied. The termination criterion most commonly used is thresholding of the value of the distance.

It is worth noticing, though, that in our case, where the input space dimensions are large, the Euclidean distance is thought to be the best distance measure used (Yager, 2000). Still, this is not always the case, owing to the nature of the individual features; consequently a selection of meaningful features needs to be performed, prior to calculating the distance (Wallace and Stamou, 2002). Moreover, one feature might be more important than others, while all of the features are useful, each one to its own degree. In this work we tackle weighting of features based on the following principles:

- We expect elements of a given meaningful set to have random distances from one another according to most features, but we expect them to have small distances according to the features that relate them.

- We select meaningful features based on the nature of the specific questions of the e-questionnaires. In particular, system experts perform an initial selection of meaningful questions, restricting the input space dimensions.

- We further perform a second level filtering of the input data, based on the type of the input, leaving out answers - and thus questions - of arbitrary dimensions, such as free text input boxes of the e-questionnaires. Information collected from such answers fails out of the scope of clustering data and identifying user profiling information, being more useful for plain statistical approaches.

More formally, let $c_{1}$ and $c_{2}$ be two clusters of elements. Let also $r_{i}, i \in N_{F} r_{i}$, be the metric that compares the $i$ th feature, and $F$ the overall count of features (the dimension of the input space). A distance measure between the two clusters, when considering just the $i$ th feature, is given by:

$$
f_{i}\left(c_{1}, c_{2}\right)=\sqrt[\kappa]{\frac{\sum_{\alpha \in c_{1}, b \in c_{2}} r_{i}\left(a_{i}, b_{i}\right)^{\kappa}}{\left|c_{1}\right|\left|c_{2}\right|}}
$$

where $e_{i}$ is the $i$ th feature of element $e,|c|$ is the cardinality of cluster $c$ and $\kappa$ is a constant. The overall distance between $c_{1}$ and $c_{2}$ is calculated as:

$$
d\left(c_{1}, c_{2}\right)=\sum_{i \in N_{F}} x_{i}\left(c_{1}, c_{2}\right)^{\lambda} f_{i}\left(c_{1}, c_{2}\right)
$$


where $x_{i}$ is the degree to which $i$, and therefore $f_{i}$, is included in the soft selection of features, $i \in N_{F}$ and $\lambda$ is a constant. Based on the principle presented above, values of vector $x$ are selected through the minimisation of distance $d$, i.e.:

$$
\begin{aligned}
& x_{1}\left(c_{1}, c_{2}\right)=\frac{1}{\sum_{i}\left[f_{1}\left(c_{1}, c_{2}\right) / f_{i}\left(c_{1}, c_{2}\right)\right]^{1 / \lambda-1}} \\
& x_{i}\left(c_{1}, c_{2}\right)=x_{1}\left[\frac{f_{i}\left(c_{1}, c_{2}\right)}{f_{1}\left(c_{1}, c_{2}\right)}\right]^{1 / \lambda-1} .
\end{aligned}
$$

Of course, when $\lambda=1$ the solution is trivial and the feature that produces the smallest distance is the only one selected. The degree to which it is selected is 1 . A more detailed approach on the issue can be found in Wallace and Kollias (2003).

In the following, we present the proposed algorithm implementation with our system's data set, using the Euclidean distance as the distance measure. The clustering algorithm has been applied to a small portion of the dataset, namely a $10 \%$ of the overall system's users; it contained 100 elements/users, characterised by 44 meaningful features/questions. The overall dataset consisted of answers provided by 1000 users from eight European countries and in seven European languages, shown in Figure 12, on the same set of 44 selected features.

Figure 12 SPERO dataset languages

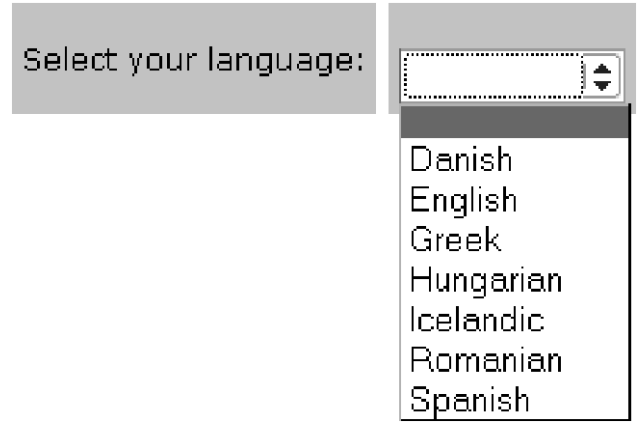

Statistical analysis and cross-correlation selection of features indicated the most important ones that were considered in the following. Although meaningful features formed a merely $25 \%$, this proved to be accurate and efficient in the process. They correspond to a set of questionnaire questions together with their possible answering options that are summarised in the following Table 1 and have been considered indicative of the profiling extraction process. Features (column 1) are grouped by the corresponding question id (column 2) of the e-questionnaire. For each verbal question description presented in the third column of Table 1, the number of associated question ids and features used in the clustering procedure are shown in the first and second columns of Table 1 respectively. 
Table 1 Feature selection

\begin{tabular}{|c|c|c|}
\hline Feature & QuestionID & Description \\
\hline 1 & 125 & $\begin{array}{l}\text { Are you a teacher dedicated to or working in special } \\
\text { education needs? }\end{array}$ \\
\hline $2,3,4$ & $126,127,128$ & Qualification/training in special education needs? \\
\hline 5 & 130 & Teaching experience \\
\hline 6 & 133 & Do you have a computer at home? \\
\hline 7 & 134 & Do you have access to the Internet from your home? \\
\hline 8 & 139 & $\begin{array}{l}\text { How often do you personally use your Internet } \\
\text { connection at home? }\end{array}$ \\
\hline $9,10,11,12,13$ & $\begin{array}{l}141,142,143,144 \\
145\end{array}$ & $\begin{array}{l}\text { For which of the following did you use the computer at } \\
\text { least once in the past month? }\end{array}$ \\
\hline $\begin{array}{l}14,15,16,17 \\
18,19,20\end{array}$ & $\begin{array}{l}147,148,149,150 \\
151,152,153\end{array}$ & $\begin{array}{l}\text { Which of the following tasks have you performed at } \\
\text { least once, without any help? }\end{array}$ \\
\hline 21 & 155 & Are there any computers in your work environment? \\
\hline 22,23 & 300,301 & $\begin{array}{l}\text { How often did you use the computer last week at the } \\
\text { school? }\end{array}$ \\
\hline 24,25 & 168,169 & $\begin{array}{l}\text { Do you have access to the internet or educational } \\
\text { software in your work environment? }\end{array}$ \\
\hline 26,27 & 174,175 & $\begin{array}{l}\text { In your teaching, how many hours a week, on average, } \\
\text { do you use the Internet or educational software with } \\
\text { your students? }\end{array}$ \\
\hline 28,29 & 176,177 & $\begin{array}{l}\text { Do you use the internet for search and retrieval of } \\
\text { information relating to the needs and problems faced by } \\
\text { SEN students? }\end{array}$ \\
\hline 30,31 & 182,183 & $\begin{array}{l}\text { Do you use the internet from the school in order to find } \\
\text { additional sources of educational material? }\end{array}$ \\
\hline 32,33 & 400,401 & Do you use the internet to connect with other schools? \\
\hline $34,35,36,37$ & $261,262,264,265$ & Is your post permanent - temporary? \\
\hline $\begin{array}{l}38,39,40,41 \\
42,43\end{array}$ & $\begin{array}{l}267,268,270,271 \\
273,274\end{array}$ & Age of your students \\
\hline 44 & 275 & Area served by your school \\
\hline
\end{tabular}

The above elements belonged to three fixed profile classes, but this labelling information was not used during clustering; the labels were used, though, for the evaluation of the quality of the clustering procedure, as described in Mylonas et al. (2004b), prior to projecting the results to the whole data set. More specifically, each detected cluster was then assigned to the experts' provided class that dominated it. In the general case, identified clusters define specific interests and profiles, which do not necessarily correspond to the a priori known classes that are utilised during the first phase. These clusters are useful in producing collaborative recommendations of the e-learning content to the end users at a later stage, as described in Wallace et al. (2003). Results are shown in Tables 2-4, whereas the numbers inside parenthesis separated by commas denote the elements belonging to its one of the three profile classes in each step. 
Table 2100 users clustering results: three clusters

\begin{tabular}{lcc}
\hline Clusters & Elements & Percentage \\
\hline 1st & $(2,6,9)$ & $(11.77,35.29,52.94)$ \\
2nd & $(11,2,25)$ & $(28.95,5.26,65.79)$ \\
3rd & $(14,1,30)$ & $(31.11,2.22,66.67)$ \\
\hline
\end{tabular}

Table 3100 users clustering results: five clusters

\begin{tabular}{lcc}
\hline Clusters & Elements & Percentage \\
\hline 1st & $(3,1,7)$ & $(27.27,9.09,63.64)$ \\
2nd & $(5,1,8)$ & $(35.72,7.14,57.14)$ \\
3rd & $(5,1,13)$ & $(26.32,5.26,68.42)$ \\
4 th & $(5,9,11)$ & $(20.00,36.00,44.00)$ \\
5 th & $(11,1,19)$ & $(35.48,3.23,61.29)$ \\
\hline
\end{tabular}

Table 4100 users clustering results: nine clusters

\begin{tabular}{lcc}
\hline Clusters & Elements & Percentage \\
\hline 1st & $(1,1,4)$ & $(16.66,16.66,66.66)$ \\
2nd & $(0,1,6)$ & $(0.00,14.28,85.71)$ \\
3rd & $(4,2,5)$ & $(36.36,18.18,45.45)$ \\
4th & $(3,3,6)$ & $(25.00,25.00,50.00)$ \\
5th & $(4,2,5)$ & $(36.36,18.18,45.45)$ \\
6th & $(8,4,5)$ & $(47.05,23.52,29.41)$ \\
7 th & $(4,1,4)$ & $(44.44,11.11,44.44)$ \\
8 th & $(3,10,6)$ & $(15.78,52.63,31.57)$ \\
9 th & $(1,4,3)$ & $(12.50,50.00,37.50)$ \\
\hline
\end{tabular}

Performing the initial clustering on a mere $10 \%$ subset is not only more efficient computationally wise, it is also better in the means of quality and performance, when compared to the approach of applying the hierarchical process to the whole data set. Although clustering over this $10 \%$ of the data set resulted in different possible identifiable clusters, optimal results have been obtained for a number of nine clusters, as indicated in the following Tables 2-4, where clustering results are presented for three variations of output clusters $(3,5$ and 9$)$.

More specifically, Table 2 presents the clustering results of 100 users. The hierarchical clustering algorithm terminated by the time reached a threshold of three clusters. The first cluster comprises 17 users, namely 2 belonging to the Experts class, 6 to the Beginners class and 9 to the Advanced class. The corresponding percentage distribution clearly indicates that the 9 Advanced dominate the first cluster. The second cluster consists of 38 users: 11 Experts, 2 Beginners and 25 Advanced. Advanced are dominant in this cluster as well, whereas in terms of percentages their domination is confident (i.e., 65.79\%). Finally, the third cluster contains 45 users, 14 of whom are Experts, 1 is Beginner and 30 are Advanced. Domination of Advanced is more indicative, since a $66.67 \%$ gives them a clear advantage. 
Table 3 presents the clustering results of the same 100 users, however a new threshold of 5 clusters terminates the clustering algorithm earlier. In this case, all clusters contain lesser users, in comparison to the prior case. The first cluster consists of 11 users, 3 Experts, 1 Beginner and 7 Advanced. The corresponding percentage distribution clearly indicates that the 7 Advanced users dominate the first cluster. The same applies to the second cluster with 14 users as well, where 5 users belong to the Experts, 1 user is Beginner and 8 users are in the intermediate state, i.e., Advanced. Third, fourth and fifth clusters are all resulting in the supremacy of Advanced; third cluster containing 19 users (distributed accordingly to 5 Experts, 1 Beginner and 13 Advanced), fourth cluster containing 25 users (5 Experts, 9 Beginners and 11 Advanced) and fifth cluster containing 31 users (11 Experts, 1 Beginner and 19 Advanced).

Continuing in Table 4, the results of the clustering step demonstrate the clear trend underlying in the system's input data: users are characterised by intermediate ICT skills and expertise. This observation is extremely evident in the third column of Table 4, which indicates clearly that most users of the system belong to the static, intermediate Advanced profile. The first two clusters identified by our algorithm are unambiguously dominated by the third profile class, i.e., Advanced. Additionally, clusters 3, 4 and 5 indicate a clear majority of the same third class in their elements as well. Consequently, 5 out of 9 clusters (55.55\%) are indicating a clear advantage of the Advanced. Moreover, cluster 7 acts as an intermediary between Advanced and Experts, as it illustrates a draw in the elements between those two profile classes. Clusters 8 and 9 are dominated by the Beginner profile class, whereas cluster 6 forms a solid representative of the Experts.

The above-illustrated clustering approach forms the basic procedure, with the aid of which each SPERO end user is automatically categorised to a specific profile class that characterises his behaviour and his future interests and choices within the system. Additionally, each identified cluster is related to replies and specific comments of the e-questionnaires. We used clustering results to classify the responses of users to specific parts of the e-questionnaires, deriving information based on the users' profiles. The automatic extraction of clusters during the SPERO project provided experts with the ability to distinguish the responses of the clustered users with respect to more specific profiles. More specifically, we combined profiling information from different parts with respect to the users input, a statistical analysis of which is provided in Figure 13, as well as analysed results belonging to different parts of the e-questionnaires, as illustrated in Figure 14.

The work proposed here could also be combined with neural network machine learning techniques. For instance, another work of ours described in Wallace et al. (2003) uses clustering to initialise a three-layer neural network classifier that contains personalisation information for extracting the local interest of users. However, this is not considered to be the main focus of the current paper, which focuses on utilisation of clustering towards providing content collaborative recommendations to the end users. According to the cluster to which each user belongs, educational content, appropriately selected by the system's experts, is offered to him. Because of flexibility and protection of crucial personal data reasons, the step of user characterisation is only provided as an added value characteristic to the users that are willing to use it. Suitable verification procedures ensure that content offering filtering features is only enabled according to each end user's will. 
Figure 13 Learning resources linked to profile categories

\section{Teacher Questionnaire}

\begin{tabular}{|c|c|c|c|c|c|}
\hline \multicolumn{6}{|c|}{ A. Pedagogical Utilisation of Information Technologies } \\
\hline$\#$ & Profile & \multicolumn{4}{|c|}{ Color } \\
\hline 1. & \multicolumn{5}{|l|}{ Beginner } \\
\hline 2. & \multicolumn{5}{|l|}{ Advanced } \\
\hline 3. & \multicolumn{5}{|l|}{ Expert } \\
\hline 4. & \multicolumn{5}{|l|}{ Unspecified } \\
\hline \multicolumn{6}{|c|}{$\begin{array}{l}\text { A.1.Changes in the daily activities of the teaching staff due to the use of ICT. } \\
\text { A.1.1. The widespread use of ICT as a teaching tool in school will lead to a change in some aspects of daily } \\
\text { teaching practice. Please, indicate which: }\end{array}$} \\
\hline & & \multicolumn{2}{|c|}{ Mostly } & \multicolumn{2}{|c|}{ Rarely } \\
\hline \multicolumn{2}{|c|}{ A change of attitude of the teaching staff will be necessary } & 7 & $5.74 \%$ & 0 & $10 \%$ \\
\hline & & 0 & $10 \%$ & 0 & $10 \%$ \\
\hline & & 11 & $\square 9.02 \%$ & 2 & \| $1.64 \%$ \\
\hline & & 6 & $4.92 \%$ & 3 & $\begin{array}{l}\square, 46 \% \\
\end{array}$ \\
\hline
\end{tabular}

Figure 14 Combined profiling information based on clustering results

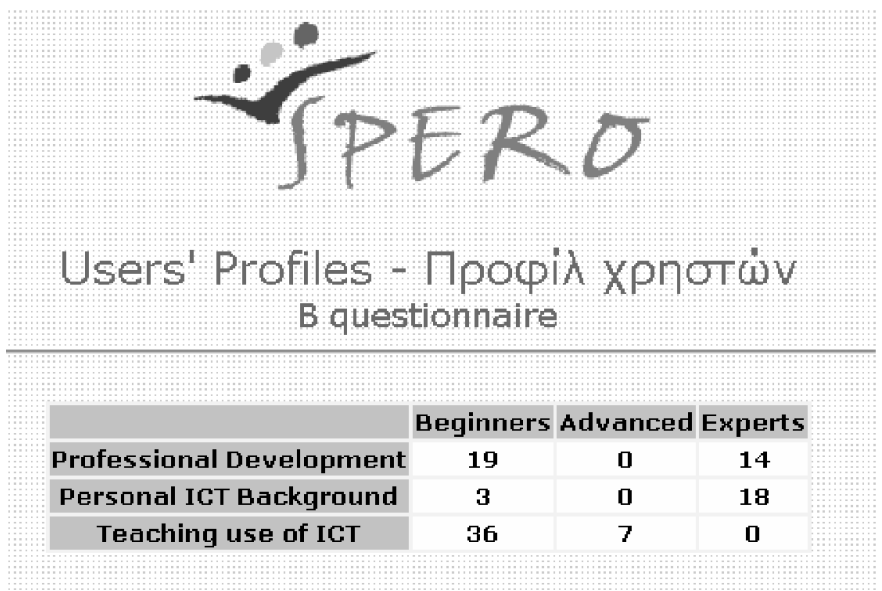

\section{Content adaptation and user tracking}

The SPERO system software forms an integrated, web-based learning portal, designed and implemented according to well-known learner-friendly solutions and flexible e-learning software applications (WebCT Campus Edition). When system's users visit the SPERO portal, validation against the system user database is performed. Subsequently, they are called to answer the e-questionnaires in order to automatically establish their user profile based on the intelligent clustering techniques presented in the 
previous sections. This automatic profile extraction provides the extremely useful and fully personalised information needed. Learning resources have been linked up to each profile category that has been defined during the profile extraction process and are illustrated in Figure 15. The set of e-courses appropriate for each of the identified groups of user profiles is selected by the group of system's experts.

Figure 15 Learning resources linked to profile categories

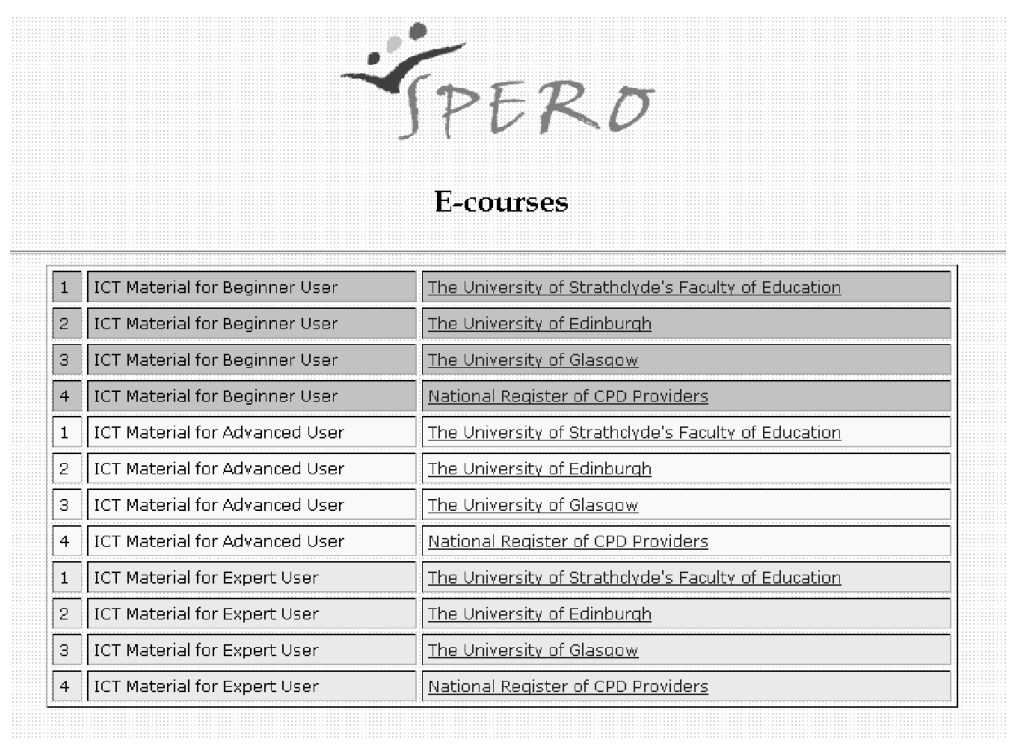

SPERO's content offering contains links to educational content, separated into various sectors and providing services, like: Courses Catalogue, Announcement Service, Search Service, E-mail Service, Upload Files and Help Service. In particular, the main menu of the SPERO portal contains links to the following sectors/services:

- Courses catalogue. It contains the titles, as well as a small textual description of one or more e-learning courses, that learners may take. An intelligent module takes over the selection of e-courses, according to user preferences and profiles, as well as their usage history. A small overview for each e-course is provided, demonstrating its main topics and concepts. A small notion of a selected e-course listing is presented in Figure 16.

- Announcement service. This service provides a bulletin board where topics about e-courses or other educational subjects are published. Relative documents, regarding e-courses outlines and requirements are posted herein. Students' and teachers' messages are presented in a threaded view layout.

- Search service. It provides a search environment to facilitate information and educational materials retrievals from SPERO site, e.g., members, school units, e-lessons, e-books, e-lectures, exercises, 'live' educational content broadcasts, etc.

- $\quad$ E-mail service. SPERO users are able to send and receive e-mails through the SPERO system. 
- Upload files. Learners have their own personal space where they can store their own material to which other learners may or may not have access to. Several levels of authorisation access are implemented.

- Help service. Analytical description of the usage and tasks of SPERO menu choices. It provides information about library links and online resources outside the SPERO system and answers general Frequently Asked Questions.

Figure 16 Personalised e-course listing sample

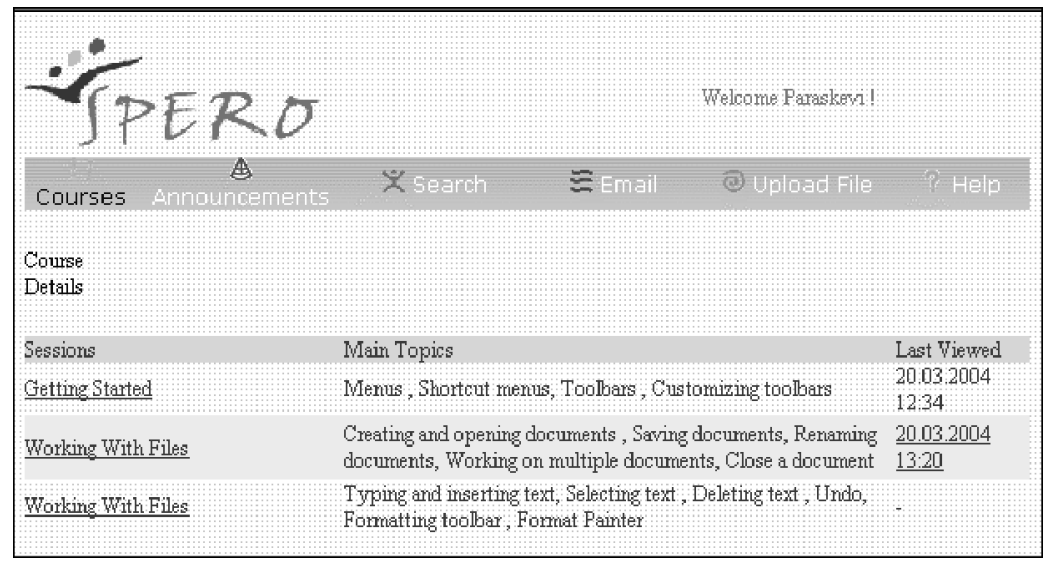

In order to improve the ICT level of learners, different e-courses are also designed and implemented. Indicatively, groups of e-courses characterised by increasing difficulty and strong topic relativity are possible, such as the following chain of e-courses: Introduction to Information's Technologies, Introduction to Operating Systems, Presentation and usage of Office and Educational software, Introduction and Usage of Internet. Each group is characterised by the following aspects:

- Group 1. Introduction to Information Technologies (definition of data, bit, byte, presentation of hardware components, presentation of type of software).

- Group 2. Presentation and usage of operating systems.

- $\quad$ Group 3. Usage of text editor, software for work sheets, software for creation a presentation, educational software.

- Group 4. Usage of Internet (explorer in a browser, search machines, sending and receiving e-mails, access to a news group, access to a chat room).

The offered e-courses correspond to the available SPERO user profiles obtained previously. For instance, e-courses for a learner, identified by the system as an 'Expert', are depicted in Figure 17. This is ensured by an intelligent user tracking mechanism. This mechanism is based on each user individual session, the starts and stops of which are signalled by the time the user enters and leaves the SPERO portal respectively. Session information, along with validation and user access rights is stored in the 'userDetails' part of the static user profile. It provides a robust and reliable method to ensure independency amongst the system's users and efficiency of the whole user profiling-based content offering. As an illustrative example, consider that students who 
receive an e-course are tracked throughout the SPERO system and their behaviour is observed and tracked internally. The overall procedure is transparent and provides the main source of feedback for the users' future system (e-courses and material) selections. Additionally, learners' interaction is periodically monitored to allow dynamically change of their user profiles, according to their improvement of ICT skills and skilfulness. For instance, if a user studies, then it is foreseeable that he or she will improve his or her profile. Towards that scope, it is possible for the learner to re-enter the SPERO portal and provide new data input for the e-questionnaires. As soon as this is done, the system, based on his or hers unique id, monitors the user's progress and changes his or hers ratings and corresponding content offering. This leaves an option for the learner that the system can provide and sets him or her on a specific personalised track.

Figure 17 E-courses list for experts learners

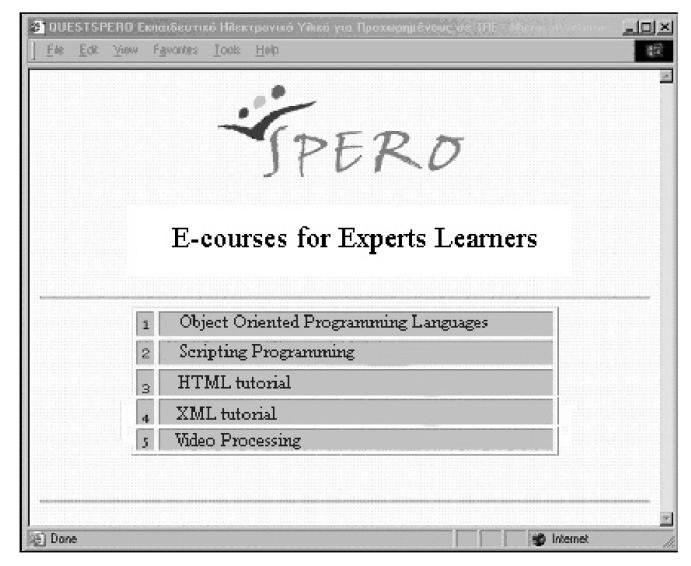

\section{Conclusions and future work}

Current approach to e-learning applications forms an integrated, state-of-the-art system that is able to identify its individual users. It extends work performed on precise, high-level personalisation algorithms. The system internally utilises personalisation techniques towards profiling extraction, introducing a novel conjunction of static and dynamic profiling mechanisms. It was also our intension to really focus on acquired representative results of this work. For more e-learning type of results the reader is encouraged to visit SPERO's online applications. Moreover, the main research effort of this paper was to successfully introduce and apply clustering techniques in the process of user profiling and provide collaborative recommendations of e-learning content to the end users.

A major area of future research for this work is the utilisation of a fuzzy relational knowledge representation model in the learners' profile weight estimation process. Our findings so far indicate, that such a combination between semantic and statistical information is possible and will have very interesting results, regarding the personalisation of the educational content offered to the end-users. Additionally, the work proposed herein could also be enriched by combining it with neural network machine learning techniques. 
This work is part of our ongoing efforts in the field of designing and implementing an integrated, fully automated e-learning portal system. The main focus is given to the personalisation aspects of the system's user handling and educational content offering. Possible future work includes better selection of the clustering algorithm threshold criteria and possible increase of the static profiles categories. Moreover, the system's e-questionnaires are susceptible to evaluation and improvements, as well as an increase in the number of participants in the e-surveys is viable. The overall proposed architecture of SPERO could be easily adapted to other e-learning schemes, mainly owing to its robustness and entities clarity.

\section{Acknowledgement}

This work has been partially funded by the "SPERO: TeleInformatics System for Continuous Collection, Processing, Diffusion of Material for Teacher Training in Special Education" European project of the 'Leonardo Da Vinci' programme, under 'Themes 5: E-Learning'.

\section{References}

Commission of European Communities (2000) Communication from the Commission: e-Learning - Designing Tomorrow's Education, Brussels.

Duda, R.O., Hart, P.E. and Stork, D.G. (2001) Pattern Classification, 2nd ed.,Wiley, New York.

IEEE LTSC (2002) P1484.1 Architecture and Reference Model, WG1 LTSA, [online] Available from: http://ieeeltsc.org/.

Karagiannidis, C., Sampson, D.G. and Cardinali, F. (2002) 'An architecture for web-based e-Learning promoting reusable adaptive educational e-content', Educational Technology and Society, Vol. 5, No. 4, pp.27-37.

Kohavi, R. and Sommerfield, D. (1995) Feature Subset Selection Using the Wrapper Model: Overfitting and Dynamic Search Space Topology, KDD-95.

Mitchell, M.T. (1997) Machine Learning, McGraw-Hill Companies, Inc., New York.

Mylonas, Ph., Tzouveli, P. and Kollias, S. (2004a) 'Towards a personalized e-learning scheme for teachers', 4th IEEE International Conference on Advanced Learning Technologies, Joensuu, Finland.

Mylonas, Ph., Wallace, M. and Kollias, S. (2004b) 'Using k-nearest neighbor and feature selection as an improvement to hierarchical clustering', 3rd Hellenic Conference on Artificial Intelligence, Samos, Greece.

Paliouras, G., Papatheodorou, C., Karkaletsis, V. and Spyropoulos, C.D. (2002) 'Discovering user communities on the internet using unsupervised machine learning techniques', Interacting with Computers, Vol. 14, No. 6, pp.761-791.

Rosenberg, J.M. (2002) E-Learning: Strategies for Delivering Knowledge in the Digital Age, McGraw-Hill, Inc., ISBN:007137809X.

Skordalakis, E. (1991) Introduction to Software Engineering, Athens.

Smith, A.S. (2001) Application of Machine Learning Algorithms in Adaptive Web-based Information Systems, CS-00-01, School of Computing Science Technical Report Series, ISSN 1462-0871.

SPERO (2001-2004) Leonardo Da Vinci programme, Themes 5: E-Learning, [online] Available from: http://www.image.ntua.gr/spero. 
Sullivan, M. (1998) 'Collaboration: teacher pedagogy, student practice', National Teaching and Learning Forum, Vol. 7, No. 5, pp.4-6.

Theodoridis, S. and Koutroumbas, K. (1999) Pattern Recognition, Academic Press, San Diego.

Varenne, H. (2001) On Pedagogical Authority: One Teacher's Choice, [online]. Available from: http://varenne.tc.columbia.edu/hv/pol/pedagog_author.html.

Wallace, M. and Kollias, S. (2003) 'Soft attribute selection for hierarchical clustering in high dimensions', International Fuzzy Systems Association World Congress (IFSA), Istanbul, Turkey.

Wallace, M. and Stamou, G. (2002) 'Towards a context aware mining of user interests for consumption of multimedia documents', IEEE International Conference on Multimedia and Expo (ICME), Lausanne, Switzerland.

Wallace, M., Karpouzis, K., Stamou, G., Moschovitis, G., Schizas, C. and Kollias, S. (2003) 'The electronic road: personalized content browsing', IEEE Multimedia, Multimedia Content Modeling and Personalization, Vol. 10, No. 4, pp.49-59.

Yager, R.R. (2000) 'Intelligent control of the hierarchical agglomerative clustering process', IEEE Transactions on Systems, Man and Cybernetics, Vol. Part B 30, No. 6, pp.835-845.

\section{Websites}

SPERO, E-questionnaires Web Application, Available from: http://www.image.ntua.gr/questspero. SPERO, Profiles Web Application, Available from: http://www.image.ntua.gr/statspero/profiles. SPERO, Statistics Web Application, Available from: http://www.image.ntua.gr/statsspero. WebCT Campus Edition: Course Management System, Available from: http://www.webct.com/. 